\title{
Variant Curation is Crucial to Claim Digenic Inheritance in Juvenile Open Angle Glaucoma
}

\author{
Kok-Siong Poon ${ }^{1}$ Karen Mei-Ling $\operatorname{Tan}^{1}$ \\ ${ }^{1}$ Department of Laboratory Medicine, National University Hospital \\ Singapore, Singapore \\ Glob Med Genet 2022;9:54-55.
}

Address for correspondence Kok-Siong Poon, Department of Laboratory Medicine, National University Hospital, 5 Lower Kent Ridge Rd, Singapore 119074 (e-mail: kok_siong_poon@nuhs.edu.sg).
The genetics of juvenile open angle glaucoma (JOAG) is heterogeneous in association with multiple loci. ${ }^{1-4} \mathrm{~A}$ recent case report described a family in which multiple members were affected by JOAG with autosomal dominant (AD) inheritance. ${ }^{5}$ In the study, the causative genetic variants were first elucidated by whole exome sequencing in the proband and her affected father, and were further confirmed by targeted Sanger sequencing in her two siblings with ocular hypertension. Pathogenic variants in the MYOC gene encoding myocilin is the most common cause of AD form of JOAG. ${ }^{6,7}$ In this family, the MYOC p.Pro370Leu variant, which is a known pathogenic variant, ${ }^{8,9}$ was found in all affected family members. An additional missense variant, p. Pro432Leu in the LTBP2 gene, was also observed to co-segregate with MYOC p.Pro370Leu. Interestingly, digenic inheritance of the MYOC and LTBP2 genes located at $1 \mathrm{q} 24.3$ and $14 \mathrm{q} 24.3$, respectively, was proposed by the authors to delineate the genetic cause of JOAG in this family.

Suggestion of digenic inheritance in JOAG is not new. A heterozygous variant in CYP1B1, which is known to cause an autosomal recessive (AR) form of JOAG, ${ }^{10}$ was reported as a modifier gene when inherited together with a MYOC pathogenic variant, predisposing the affected family members to earlier disease onset. ${ }^{11}$ Similarly, biallelic variants in the LTBP2 gene cause an AR form of JOAG. ${ }^{12}$ The authors postulated that MYOC p.Pro370Leu might be necessary for disease manifestation in one of the healthy family members who only carries heterozygous LTBP2 p.Pro432Leu. It was also hypothesized that with LTBP2 p.Pro432Leu alone, the carrier may have late disease onset since manifestation of JOAG can be progressive. When coinherited with MYOC p.Pro370Leu, the LTBP2 p.Pro432Leu was also deduced to act as a modifier allele instead of being disease-causing on its own.

Digenic inheritance occurs in a disease context when variant genotypes at two loci explain the phenotypes of some patients more clearly than the genotypes of one locus alone. ${ }^{13}$
While the MYOC p.Pro370Leu variant in comparison with other MYOC variants is associated with early onset, severe disease, and having a penetrance of more than $75 \%$ by the age of 25, the authors argue that the family members with both the MYOC and LTBP2 variants have a penetrance of $100 \%$ by the age of 15 suggesting the LTBP2 p.Pro432leu enhances the pathogenicity of the MYOC variant. The evidence of pathogenicity of the LTBP2 variant was substantiated by the authors based on computational prediction by DEOGEN-2 and FATHMM-MKL although other predictors including SIFT and PolyPhen-2 did not predict it to be deleterious. These postulations prompted us to further curate the LTBP2 p.Pro432Leu variant to classify its pathogenicity.

Although classified as a disease mutation in Human Gene Mutation Database Professional 2020.4, ${ }^{14}$ Abouelhoda et al found LTBP2 p.Pro432Leu in homozygosity in normal individuals from Saudi Arabian populations enriched for homozygosity due to inbreeding. ${ }^{15}$ The variant has a relatively high minor allele frequency (2\%) in the Indian ethnicities according to 1,000 genomes project phase III. The latest classification in ClinVar interpreted this variant with conflicting interpretations of pathogenicity, which are aggregated from four submissions including two "benign" and two "uncertain significance". Finally, we applied the American College of Medical Genetics and Genomics/Association for Molecular Pathology criteria ${ }^{16}$ using VarSome version 9.2.0 ${ }^{17}$ and found that it was predicted to be "likely benign" since only PM2, BP1, and BP4 criteria are met.

Collectively, the clinical significance of p.Pro432Leu variant remains unclear. In view of the conflicting evidence, the involvement of LTBP2 in digenic inheritance of JOAG warrants further investigation. Reporting additional variants with conflicting interpretations of pathogenicity may complicate genetic counseling or raise unnecessary anxiety in the carriers of this variant on the risk of developing genetic eye diseases. received

June 17, 2021

accepted after revision

July 9,2021

published online

August 19, 2021
DOI https://doi.org/

$10.1055 / \mathrm{s}-0041-1733963$.

ISSN 2699-9404. (c) 2021. The Author(s).

This is an open access article published by Thieme under the terms of the Creative Commons Attribution License, permitting unrestricted use, distribution, and reproduction so long as the original work is properly cited. (https://creativecommons.org/licenses/by/4.0/)

Georg Thieme Verlag KG, Rüdigerstraße 14, 70469 Stuttgart, Germany 


\section{Funding}

None.

\section{Conflict of Interest \\ None declared.}

\section{References}

1 Fan BJ, Leung DY, Wang DY, et al. Novel myocilin mutation in a Chinese family with juvenile-onset open-angle glaucoma. Arch Ophthalmol 2006;124(01):102-106

2 Wang DY, Fan BJ, Chua JK, et al. A genome-wide scan maps a novel juvenile-onset primary open-angle glaucoma locus to $15 q$. Invest Ophthalmol Vis Sci 2006;47(12):5315-5321

3 Gupta V, Somarajan BI, Gupta S, et al. The inheritance of juvenile onset primary open angle glaucoma. Clin Genet 2017;92(02):134-142

4 Turalba AV, Chen TC. Clinical and genetic characteristics of primary juvenile-onset open-angle glaucoma (JOAG). Semin Ophthalmol 2008;23(01):19-25

5 Somarajan BI, Gupta S, Mahalingam K, Azmira K, Gupta V. Digenic inheritance in juvenile open-angle glaucoma. J Pediatr Genet 2021. Doi: $10.1055 / \mathrm{s}-0040-1722213$

6 Fingert JH. Primary open-angle glaucoma genes. Eye (Lond) 2011; 25(05):587-595

7 Wiggs JL, Pasquale LR. Genetics of glaucoma. Hum Mol Genet 2017;26(R1):R21-R27

8 Adam MF, Belmouden A, Binisti P, et al. Recurrent mutations in a single exon encoding the evolutionarily conserved olfactomedinhomology domain of TIGR in familial open-angle glaucoma. Hum Mol Genet 1997;6(12):2091-2097
9 Suzuki Y, Shirato S, Taniguchi F, Ohara K, Nishimaki K, Ohta S. Mutations in the TIGR gene in familial primary open-angle glaucoma in Japan. Am J Hum Genet 1997;61(05):1202-1204

10 Souzeau E, Hayes M, Zhou T, et al. Occurrence of CYP1B1 mutations in juvenile open-angle glaucoma with advanced visual field loss. JAMA Ophthalmol 2015;133(07):826-833

11 Vincent AL, Billingsley G, Buys Y, et al. Digenic inheritance of early-onset glaucoma: CYP1B1, a potential modifier gene. Am J Hum Genet 2002;70(02):448-460

12 Saeedi O, Yousaf S, Tsai J, Palmer K, Riazuddin S, Ahmed ZM. Delineation of novel compound heterozygous variants in LTBP2 associated with juvenile open angle glaucoma. Genes (Basel) 2018;9(11):527

13 Schäffer AA. Digenic inheritance in medical genetics. J Med Genet 2013;50(10):641-652

14 Stenson PD, Mort M, Ball EV, et al. The human gene mutation database: towards a comprehensive repository of inherited mutation data for medical research, genetic diagnosis and next-generation sequencing studies. Hum Genet 2017;136(06):665-677

15 Abouelhoda M, Faquih T, El-Kalioby M, Alkuraya FS. Revisiting the morbid genome of Mendelian disorders. Genome Biol 2016;17 (01):235

16 Richards S, Aziz N, Bale S, et al; ACMG Laboratory Quality Assurance Committee. Standards and guidelines for the interpretation of sequence variants: a joint consensus recommendation of the American College of Medical Genetics and Genomics and the Association for Molecular Pathology. Genet Med 2015;17(05): 405-424

17 Kopanos C, Tsiolkas V, Kouris A, et al. VarSome: the human genomic variant search engine. Bioinformatics 2019;35(11): 1978-1980 